\title{
Functional Popliteal Artery Entrapment Syndrome: A Review of the Anatomy and Pathophysiology
}

\author{
Matthew Hislop ${ }^{1^{*}}$, Dominic Kennedy ${ }^{2}$ and Sanjay Dhupelia ${ }^{2}$ \\ ${ }^{1}$ Brisbane Sports and Exercise Medicine Specialists, Australia \\ ${ }^{2}$ Queensland X-Ray, Greenslopes Private Hospital, Brisbane, Australia
}

"Corresponding author: Matthew Hislop, Brisbane Sports and Exercise Medicine Specialists 87 Riding Road, Hawthorne, Queensland 4171 , Australia, Tel: +61 7 38990659; Fax: +61738993135; Email: info@bsems.com.au

Copyright: (c) 2014 Hislop M, et al. This is an open-access article distributed under the terms of the Creative Commons Attribution License, which permits unrestricted use, distribution, and reproduction in any medium, provided the original author and source are credited.

Received date: April 06, 2014; Accepted date: May 29, 2014; Published date: June 4, 2014

\begin{abstract}
Objectives: This paper reviews the knowledge regarding the embryogenesis and anatomy of the popliteal fossa with the aim of determining which variations predispose towards exertional leg pain and Functional Popliteal Artery Entrapment Syndrome (PAES) in particular.
\end{abstract}

Methods: Current literature review and synopsis of published knowledge is presented. Ethics approval was granted for this study by the Greenslopes Research and Ethics Committee.

Results: Functional PAES is thought to be more common than previously recognized. Four subgroups of clinical presentation were found, depending on anatomical variation in the popliteal fossa: Anatomical PAES; Functional PAES; Asymptomatic Occluders and Asymptomatic Non-occluders. Features predisposing towards Functional PAES include a more lateralized medial head of gastrocnemius and a lower percentage of maximal force of plantarflexion required to cause occlusion of the artery.

Conclusions: Functional PAES may be responsible for a large number of previously unrecognized cases of exertional leg pain, and an understanding of how it develops is important. In understanding the anatomical variations present in the popliteal fossa, one can classify which sub-group the patient belongs to, and how much this predisposes towards the development of exertional leg pain. The necessity for treatment, as well as where interventions should be targeted, can be determined by coupling these investigation findings with clinical features of claudicant leg pain.

Keywords: Popliteal artery; Claudication; Anatomic variation; Pathophysiology

\section{Introduction}

Exertional leg pain is a cause of significant morbidity and limitation of activity to athletes and those who exercise. Popliteal artery entrapment syndrome (PAES) is an important and often over looked differential diagnosis in the assessment of exertional leg pain. PAES is defined as a group of conditions in which compression of the popliteal artery, popliteal vein, and tibial nerve (singly or in combination) in the popliteal fossa by surrounding musculoskeletal structures occurs to a degree sufficient to cause vascular and neurogenic symptoms [1].

PAES can be further divided into two groups, anatomical and functional. In the case of anatomical PAES, there is a clearly defined anatomical lesion that directly leads to entrapment and subsequent occlusion of the popliteal artery. The second and larger subgroup of anatomically 'normal' or functional PAES is particularly poorly understood [2]. In functional PAES there is evidence of popliteal artery occlusion and subsequent claudicant symptoms, but no defined lesion can be found that directly causes the occlusion. Rather as will be seen, there are possible anatomical variations that lead to 'crowding' of the popliteal fossa, and subsequent arterial occlusion.
The Popliteal Vascular Entrapment Forum in 1998 came up with a classification system for types of PAES and this is demonstrated in Figure $1[1,3]$. Complicating this further is the fact that there are a large number of patients who are seemingly asymptomatic but who can occlude their popliteal artery by placing the leg into sustained provocative positions (typically a combination of knee extension and plantarflexion) [4,5]. What is unknown is whether these asymptomatic patients are at risk of developing symptoms and arterial pathology with time [6].

This paper will endeavour to describe the anatomy and pathophysiology leading to the development of both anatomical and functional PAES, including the embryogenesis of the popliteal fossa, and anatomical variations which predispose to the development of PAES.

\section{Incidence}

The true incidence of functional PAES is unknown, although it is described in the literature as being rare $[3,7]$. However the incidence may be greater than previously recognized, and it is possible that this condition is under reported [6]. Subclinical symptoms may be misdiagnosed, and the condition is often confused or in fact may coexist with chronic exertional compartment syndrome (CECS), with which it shares many clinical features [2]. 
As mentioned, there are a significant proportion of asymptomatic individuals who can occlude the popliteal artery with sustained provocative positioning. The reported prevalence of popliteal artery occlusion in asymptomatic individuals detected with duplex ultrasound imaging varied between studies from $7.1 \%$ to $80 \%$ and some authors describe the condition as a 'physiological phenomenon' [3]. The high incidence of occlusion in healthy individuals further highlights the need for a clear protocol to examine and investigate the patients [3].

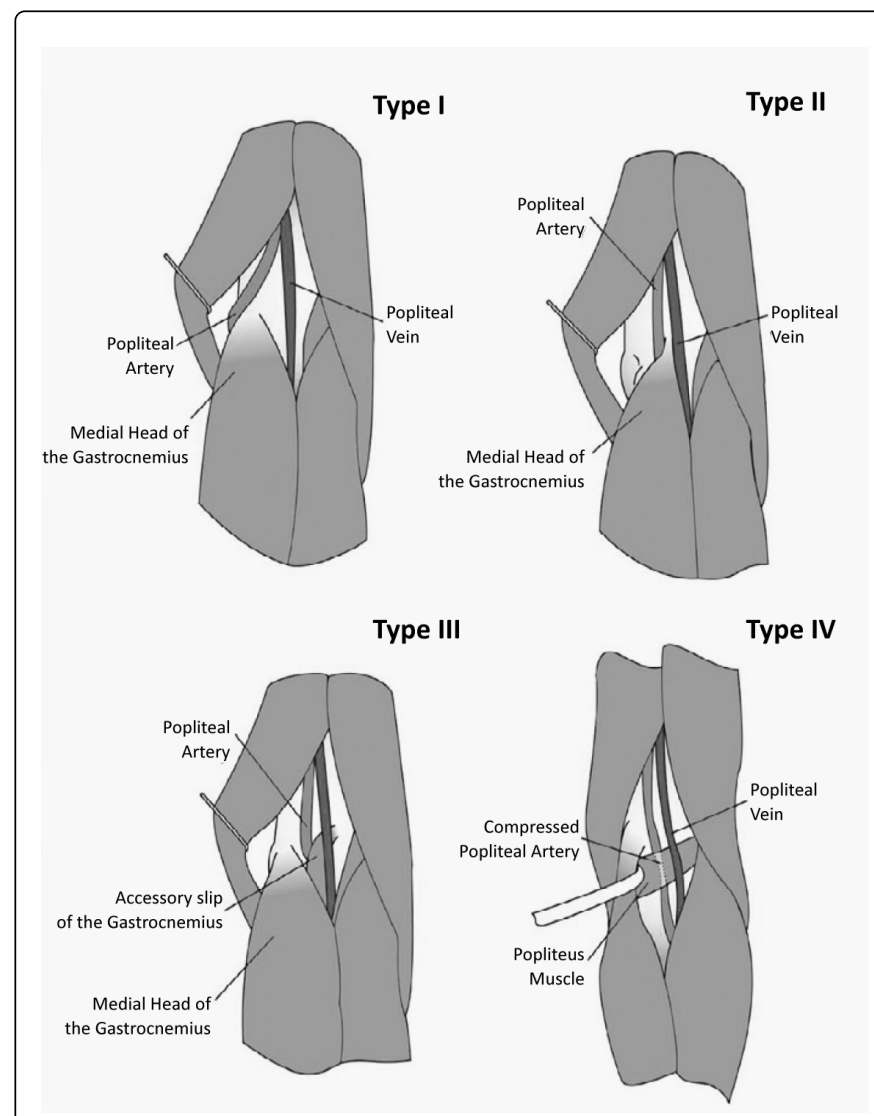

Figure 1: Popliteal Vascular Entrapment Forum Classification System for PAES. Missing are Type V (Primarily Venous Entrapment), Type VI (Other types of entrapment) and Type F (Functional Entrapment). (Figures reproduced from Pillai with permission.[1])

\section{Embryological development}

The embryological development of the popliteal artery is complex, but an understanding helps appreciate the differing types of popliteal artery entrapment. Levien et al. [6], provides an excellent summary of the embryogenesis of the popliteal fossa, and a synopsis is provided here and in Figure 2. During development, the medial head of gastrocnemius muscle (MHGM) migrates from an initial lateral position across the back of the knee to eventually attach to the posterior surface of the medial femoral condyle. At the same time there is rearrangement of the arterial structures forming the definitive popliteal artery [6].

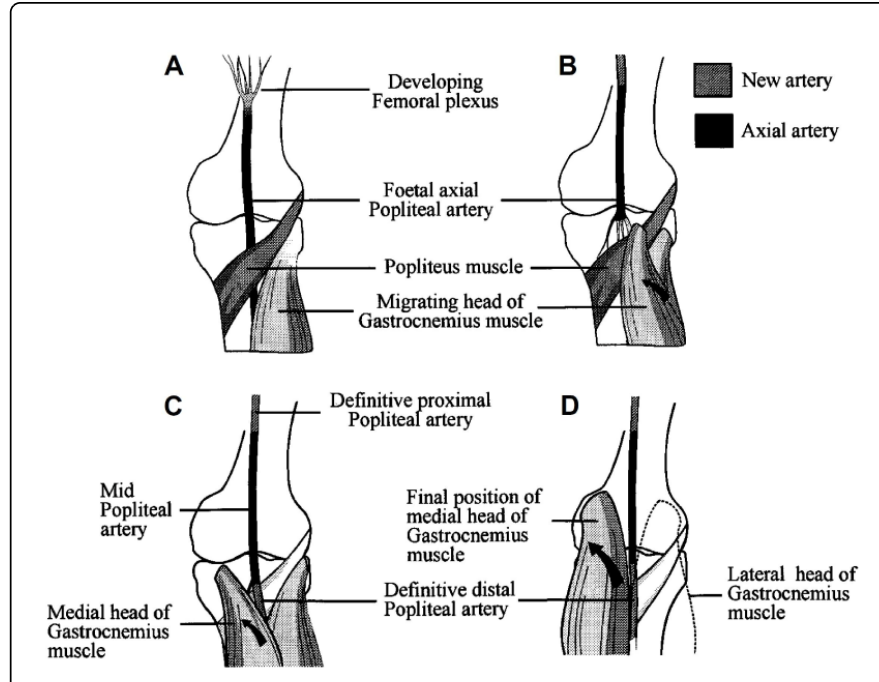

Figure 2: Representation of the Embryogenesis of the Popliteal Artery and Gastrocnemius muscle. The medial head of the gastrocnemius is seen to migrate laterally across the femoral condyles, eventually resting on the medial femoral condyle. As this happens the original axial artery deep to popliteus disappears, and is replaced by the new artery now superficial to popliteus. (Figures reproduced from Levien et al. with permission.[6])

The embryologic distal popliteal artery initially runs deep to the popliteus muscle. During development this part disappears, and a new distal part forms, superficial to popliteus after the MHGM has migrated medially [6]. If the definitive distal popliteal artery forms before or during the migration of the medial head, the newly formed artery may be swept medially with the migrating muscle. This can lead to Types I-III variations of anatomical popliteal artery entrapment. Type IV occurs when the axial artery persists as the definitive distal popliteal artery, running deep to popliteus. Any type of entrapment may involve the popliteal vein (termed Type V Entrapment) or the tibial nerves [6].

In many cases the MHGM does not migrate all the way across the popliteal fossa, and the commonest anomalies involve the incomplete or delayed migration. The final, more lateral 'resting place' of the MHGM can then cause displacement of the popliteal artery as it runs between the condyles. This "competition for space" between the primitive neurovascular bundle and various migrating muscles potentially predisposes to functional PAES. Contraction of the bulky, powerful MHGM within a confined space bordered by strong fascia and solid bone can result in forceful compression of the adjacent neurovascular bundle [1]. In these cases the anatomy in the popliteal fossa is 'normal' but variations in the position of the MHGM likely predispose to functional PAES.

\section{Muscle variation in the popliteal fossa: The 4 groups and their features}

It is apparent that there are 4 potential groups of patients, representing a spectrum from normal anatomy and asymptomatic, to frank anatomical occlusion of the popliteal artery. These 4 groups are determined by anatomical variations, and the eventual position of the MHGM as it migrates during embryological development. 
Asymptomatic Non-occluders: These are 'normal' patients with no evidence of arterial occlusion. In these patients the MHGM is more likely to have attached to its classically described resting spot on the back of the medial femoral condyle, with little or no bulk sitting in the popliteal fossa. This group most likely forms $50 \%$ or less of all patients [6].

Asymptomatic occluders: This group presents as a diagnostic dilemma, and there is the risk of 'over calling' a diagnosis of functional PAES. These patients do not complain of claudicant leg pain, and yet can occlude flow through the popliteal artery when assessed on Doppler ultrasound. There is likely some form of anatomical predisposition, especially incomplete migration of the MHGM so that there is crowding of the intercondylar notch [6]. MRI studies have confirmed that individuals who can occlude their popliteal artery with forceful plantarflexion had a more extensive MHGM attachment towards the midline of the bone just above the medial condyle as well as more extensive attachment within the intercondylar notch [8]. Figure 3 demonstrates a MRI of this type of anatomical 'crowding'. However in individuals where this anomaly is not 'extreme' then it is proposed this occlusion occurs with little risk of ongoing injury. The numbers of patients in this group is estimated at $20-85 \%$ of legs $[3,4,6]$.

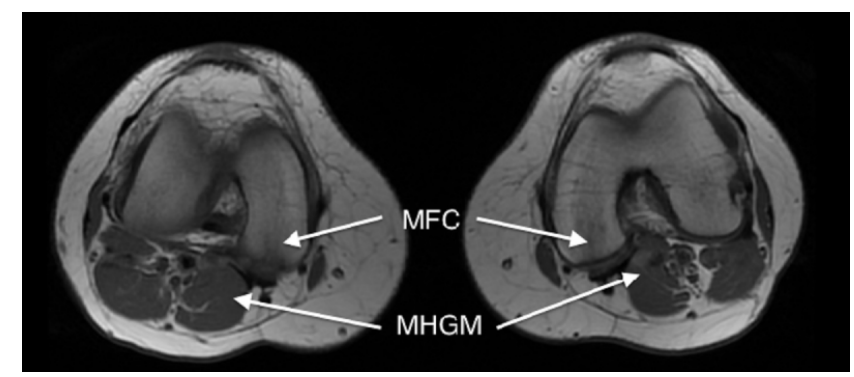

Figure 3: Axial MRI images of the popliteal region of the knee. The medial head of gastrocnemius muscle (MHGM) is seen to arise from a more lateral position off the posterior aspect of the medial femoral condyle (MFC) leading to 'crowding' of the popliteal vessels.

The ability to occlude the popliteal artery with provocative manoeuvres seems to be independent of sex or the level of physical conditioning. Asymptomatic occluders tend to occlude their popliteal artery bilaterally [4]. Active knee extension and ankle plantarflexion seems to be the usual position that will provoke occlusion [4,5]. Given the incidence of occlusion in healthy individuals, reliance solely on radiologic demonstration of vascular occlusion for diagnosis of PAES in symptomatic patients is likely to yield a significant number of falsepositive results $[3,6]$. Likewise 'prophylactic' surgical release of asymptomatic occluders is felt to be inappropriate [9].

Functional PAES: This group represents a subset of patients with no defined anatomical abnormalities, yet who can develop severe reproducible symptoms of vascular entrapment which is cured by operative exploration of the popliteal space [4]. The prevalence is unknown although this group is felt to be far more common than anatomical PAES $[5,6,9]$. The progression of disease is also unknown but it may be that these patients develop arterial injury more gradually with onset of more significant symptoms later in life. Some authors caution that repetitive microtrauma leads to cumulative arterial damage, with possible development of disruption of the internal elastic lamina, destruction of smooth muscles, and proliferation of connective tissue. Progression of local disease may lead to intraluminal stenosis, post-stenotic dilatation, and aneurysm formation $[1,9,10]$. However, given the high incidence of popliteal artery occlusion in otherwise asymptomatic individuals with provocative manoeuvres, it is indicated that only those functional PAES sufferers with significant symptoms be offered intervention, particularly in the form of surgery [6].

The cause of pain in functional PAES remains controversial, with some authors suggesting a clear ischaemic role [3], while others propose that the pain is predominantly due to nerve entrapment, with subsequent pain, cramping and/or paraesthesia [9]. As opposed to anatomical PAES, these patients are thought to be younger, more commonly female, and more active [9]. Functional PAES seems to be predominantly bilateral $[4,10]$. Some authors suggest that this condition is more common in elite or high level athletes [10] although more recent studies suggest this is not the case, with untrained or sedentary individuals also able to demonstrate popliteal artery occlusion with provocative manoeuvres [1].

There are suggested to be 4 potential sites of artery entrapment in functional PAES noted on magnetic resonance imaging (MRI) during provocative testing $[4,8]$. These sites are:

- Between plantaris and the medial head of gastrocnemius muscle (MHGM)

- Between plantaris and popliteus (where occlusion seems to occur in a posterior to anterior direction)

- At the soleal sling

- Between the MHGM and the lateral femoral condyle.

We would include a fifth site of entrapment between the MHGM and lateral head of gastrocnemius (LHGM), where we can demonstrate occlusion in a side to side direction.

Anatomical PAES: These patients have confirmed anatomical entrapment of the popliteal artery and represent Types I-IV. Patients are thought to be more commonly male (70\%), older and more sedentary [9]. The prevalence is unknown although studies report between a $0.62-3.5 \%$ incidence amongst the general population [10]. The perils of not treating confirmed anatomical PAES include fairly rapid and progressive arterial intimal damage, and ultimately limb threatening ischaemic injury [3]. Early and aggressive surgical intervention is indicated for confirmed anatomical PAES, to prevent significant arterial damage $[6,8,9,11]$.

\section{Arterial variation in the popliteal fossa}

The popliteal artery itself can have considerable variation in its pattern of branching at and below the knee joint. The 'normal' pattern, which occurs in around $92 \%$ of cases, is where the anterior tibial artery is the first branch off the main trunk, below the popliteus muscle, followed by a bifurcation into the peroneal and posterior tibial arteries. Up to 75 variations of normal can occur [12], including branches above the knee joint, and hypoplastic branches. This combined with muscular entrapment will affect the pattern and severity of symptoms, and subsequent treatment options [12,13]. Therefore knowledge of the path of the popliteal artery and its branches is essential.

\section{Force of plantarflexion required to cause occlusion}

There exists a 'grey area' with cross-over between the asymptomatic occluders and functional PAES. It is likely that muscle movement 
associated with daily activity and social exercises may not generate sufficient force to occlude and injure the artery in most occluders [8].

Hoffman et al. [14], suggest that the force level of active plantarflexion required to occlude the popliteal artery is important, and may be able to differentiate functional PAES patients from asymptomatic occluders [14]. They studied a group of asymptomatic occluders and found that $88 \%$ of their subjects could occlude their popliteal artery with forceful plantarflexion, meaning that $12 \%$ would not occlude the artery, even at maximal plantarflexion force (representing asymptomatic non-occluders). In the occluding patients, the mean force necessary to occlude the vessel was $45.1+11.5 \mathrm{~kg}$ in all subjects or $70.1 \%+/-16.8 \%$ of plantarflexion maximum force. The Hoffman study had one female subject in whom occlusion occurred at substantially lower pressures compared to other subjects (12 and $13 \mathrm{~kg}$ of plantarflexion force). No anatomical lesions were discovered on MRI, and this subject possibly represents a case of functional PAES [14].

Based on this paper and our observations, we agree with the suggestion that the force of plantar flexion necessary to occlude the popliteal artery is a crucial parameter to differentiate between "physiologic" and pathologic vessel occlusion during the provocation test [14]. Seemingly most 'asymptomatic occluders' will occlude at around $70 \%$ of maximal plantarflexion force. Many studies make the diagnosis of functional PAES based on occlusion with passive plantarflexion positioning against no resistance. It is our experience that there is a cohort of symptomatic occluders that will be missed if tested against no resistance. However, it is likely that there is overlap between asymptomatic occluders and functional PAES at different levels of resistance. We propose that there will be an average (and as yet undetermined) force of plantarflexion between 0 and $70 \%$ of maximum that will be maximally sensitive and specific to discriminate between functional PAES and the asymptomatic occluders. There is need for further study to determine a 'cut-off percentage of maximum plantarflexion force that can be used to clinically to separate the two groups.

\section{Conclusion}

Functional PAES is a poorly understood condition with a confusing clinical picture and progression. An understanding of the embryogenesis and anatomical variation evident in the popliteal fossa suggests that a more laterally positioned MHGM can result in 'crowding' of the popliteal fossa. Studies suggest that patients with this anatomical predisposition can, by performing provocative manoeuvres (especially knee extension and ankle plantarflexion) occlude the popliteal artery. The percentage of asymptomatic patients who can do this is estimated between 20 and $85 \%$. Some of this group of patients will go on to develop claudicant leg symptoms, representing functional PAES. The force of plantarflexion required to cause occlusion seems important, and patients who occlude with less plantarflexion force (some even with passive positioning against no resistance) seem to meet the criteria to diagnose functional PAES. Further work is needed to determine a force of plantarflexion between $0-70 \%$ of maximum that, along with the clinical level of suspicion, can be used to differentiate asymptomatic and functional occluders. Accurate diagnosis is essential, otherwise there is a risk of labelling and unnecessarily treating asymptomatic occluders.

\section{Practical implications}

- The clinician should consider Functional PAES in their differential diagnosis of exertional leg pain, and it may be more common than previously thought.

- Embryogenesis of the popliteal fossa leads to the development a number of anatomical subgroups, some of which predispose to exertional leg pain.

- By investigating the anatomy in the popliteal fossa the clinician can confirm their clinical suspicion as to whether PAES is the cause of the leg pain

\section{Acknowledgements}

We acknowledge Queensland X-ray for their assistance in providing high quality affordable investigations to our subjects.

\section{References}

1. Pillai J (2008) A current interpretation of popliteal vascular entrapment. J Vasc Surg 48: 61S-65S.

2. Turnipseed WD (2009) Functional popliteal artery entrapment syndrome: A poorly understood and often missed diagnosis that is frequently mistreated. J Vasc Surg 49: 1189-1195.

3. Sinha S, Houghton J, Holt PJ, Thompson MM, Loftus IM, et al. (2012) Popliteal entrapment syndrome. J Vasc Surg 55: 252-262.

4. Erdoes LS, Devine JJ, Bernhard VM, Baker MR, Berman SS, et al. (1994) Popliteal vascular compression in a normal population. J Vasc Surg 20: 978-986.

5. Deshpande A, Denton M (1998) Functional popliteal entrapment syndrome. Aust N Z J Surg 68: 660-663.

6. Levien LJ, Veller MG (1999) Popliteal artery entrapment syndrome: more common than previously recognized. J Vasc Surg 30: 587-598.

7. love Jw, Whelan Tj (1965) Popliteal Artery Entrapment Syndrome. Am J Surg 109: 620-624.

8. Pillai J, Levien LJ, Haagensen M, Candy G, Cluver MD, et al. (2008) Assessment of the medial head of the gastrocnemius muscle in functional compression of the popliteal artery. J Vasc Surg 48: 1189-1196.

9. Turnipseed WD (2012) Popliteal entrapment in runners. Clin Sports Med 31: 321-328.

10. Baltopoulos P, Filippou DK, Sigala F (2004) Popliteal artery entrapment syndrome: anatomic or functional syndrome? Clin J Sport Med 14: 8-12.

11. Ozkan U, Ö̈Ÿuzkurt L, Tercan F, Pourbagher A (2008) MRI and DSA findings in popliteal artery entrapment syndrome. Diagn Interv Radiol 14: 106-110.

12. Kim D, Orron DE, Skillman JJ (1989) Surgical significance of popliteal arterial variants. A unified angiographic classification. Ann Surg 210: 776-781.

13. Mavili E, DÃ nnmez H, Kahriman G, Ã-zaÅŸlamacÄ A Ã-zcan N, et al. (2011) Popliteal artery branching patterns detected by digital subtraction angiography. Diagn Interv Radiol 17: 80-83.

14. Hoffmann U, Vetter J, Rainoni L, Leu AJ, Bollinger A (1997) Popliteal artery compression and force of active plantar flexion in young healthy volunteers. J Vasc Surg 26: 281-287. 